\title{
The Research on Social Capital of
}

\section{Industry-University-Research Institute Cooperation}

\author{
Lin Bao \\ School of Management, Xuzhou Normal University, Xuzhou 221009, China \\ E-mail: yybaolin@163.com
}

\begin{abstract}
The technological cooperation and technological innovation are not only the material and technological activities, but also the complex social process. The industry-university-research institute (IUR) cooperation cannot success without the social capital which is embedded in the cooperative members' relation network. Based on the framework of social capital Nahapiet and Ghoshal concluded, this article analyzed the social capital factors which influence realization of industry-university-research institute cooperation from the structural, relational and cognitive dimensions. And it proposed some countermeasures to promote realization of IUR cooperation in the perspective of social capital.
\end{abstract}

Keywords: Social capital, Industry-University-Research institute (IUR), Innovation

\section{Introduction}

The cooperation of industry-university-research institute (IUR) is an inevitable outcome of market economy and knowledge economy, and is a very important part in national innovation system. Although there are 30,000 scientific and technological (S\&T) achievements each year in China, only $30 \%$ of them can be transformed and only $10 \%$ can be successfully industrialized. This strongly hinders the development of the Chinese economy. With the data collection and analysis, it can be found that the Chinese and foreign scholars have begun to research the IUR cooperation in the perspective of knowledge management and knowledge alliance rather than in the perspective of surface problem and organization pattern. Because of insufficient cognition of the social network environment which influences and restricts realization of IUR cooperation, the researchers' studying methods were probably limited in technological field, and were lack of the perspective of social capital, which were mostly based on the social network. With the deepening of research on the technological cooperation and innovation, foreign scholars (Fountain, 1997; Dyer and Singly, 1998; Lane and Lubatkin, 1998) have begun to apply the social capital theory of the new economic sociology to the research on the technological cooperation and diffusion. They noted that the inter-organizational relation creates the chances of acquiring and utilization knowledge. And the interpersonal relationship connecting with organizations has great significance for the application of new technology (Rogers, 1995). Scholars have generally thought that the social capital which takes social relation network as main content, remarkably affects the technological cooperation and innovation. Therefore, the social capital will be a good analysis pattern, and it can broaden the research approach for researching IUR cooperation and innovation.

\section{Social capital analytical framework of IUR cooperation}

The foreign scholars such as Bourdieu, Coleman, Burt, Putnam, Granovetter, etc, made a great contribution to the contemporary social capital theory. Based on the studies of the predecessors, Nahapiet, Ghoshal and Tsai $(1997,1998)$ began to apply the social capital concept to the research field of management, and probed into the relationship between social capital, intellectual capital and creation of enterprises value. They defined social capital as the sum of the actual and potential resources embedded within, available through, and derived from the network of relationships possessed by an individual or social unit. Also they separated three basic dimensions of social capital: the structural, the relational and the cognitive dimensions, which provided a clear analytical framework for organization researching. The structural dimension is the whole pattern of individual relationship, emphasizes the impersonal configuration of social network, and focuses on the characteristics of network connection and network configuration, such as existence or inexistence of network connection, intenseness or weakness of network ties, density, connectivity, hierarchy, and core of network, etc. The relational dimension is the asset created and maintained by interpersonal relationship, emphasizing the personification of social network, e.g., trust and trustworthiness, norms and sanctions, obligations and expectations, and identity and identification. The cognitive dimension refers to those resources providing shared representations, interpretations, and systems of meaning among different actors, such as shared language and codes (Arrow, 1974; Cicourel, 1973; Monteverde, 1995) and shared narratives (Orr, 1990), etc. Synthesizing the research of Nahapiet, Ghoshal and Tsai, Guoyi and Zhuxi (2003) showed the analytical framework of social capital in the figure 1. 


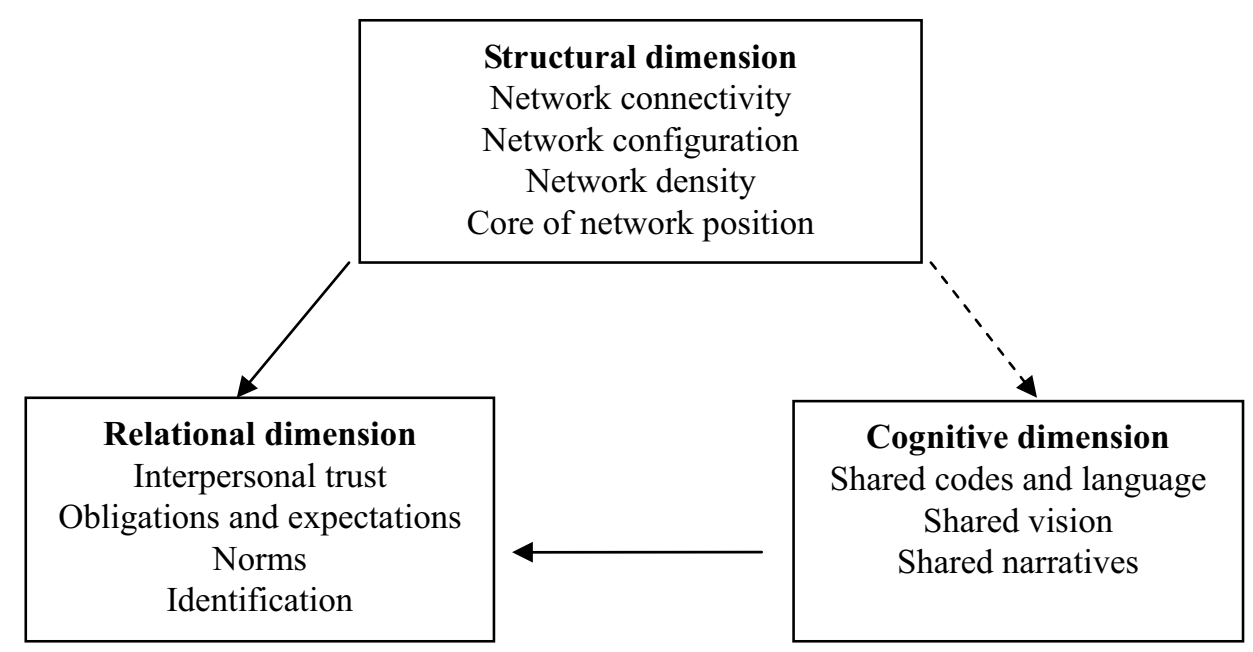

Figure 1. Social capital analytical framework

In the view of the new economic sociology, the IUR cooperation is a social process. The activities of behavior subjects are embedded in the social network, and restrained by it. The technological cooperation and technological innovation are not only the material and technological activities, but also the complex social process. It is impossible for a lot of successful realization of the IUR cooperation and innovation without the social capital which embedded in the cooperative members' relation network. The social capital can timely provide the critical resource and information for the network members; increase the association among them; promote the formation of trust; strengthen cooperative expectations; and economize supervision cost. On the other hand, with these functions, social capital of enterprise is beneficial to organization's creation, accumulation and utilization of knowledge. As the organization pattern of inter-organizational knowledge transference and management, the IUR cooperation should be analyzed in the method of the social capital through these dimensions.

\section{Analysis of social capital of IUR cooperation}

\subsection{Analysis of structural dimension of the IUR social capital}

The structural dimension of social capital mostly describes network connectivity and network configuration. The network ties of IUR cooperation refer to those relations between enterprises, universities, and research institutes. With the network ties, the universities and the research institutes provide knowledge and technology for the enterprises. At the same time, they get the market demands offered by the enterprises, which facilitate the creation of S\&T. Thus the ties in the IUR network are the important channels to obtain knowledge, technology, and information for the relevant members. There are two properties of the network connection: intenseness and weakness. The strong ties can maintain the relationship among members, facilitate communication of tacit knowledge and high quality information among enterprises (Hansen, 1999), and decrease the uncertainty of obtaining information and opportunity cost. In addition, they enhance the mutual trust with which accelerates the exchanging of information and knowledge. Therefore, the strategy alliance, joint technological creation, and co-founding enterprise entity are more and more welcome to the enterprises, universities, and institutes. On the other hand, Granovetter (1973) thought that the more strong-ties, the more similar between actors. The homogeneous information obtained from strong ties will cause the redundancy and repetition of information. In contrast, the weak ties are able to overcome these shortcomings (Granovetter, 1973). The weak ties can bring the disparate information of other group to the individual who is not belong to this group, because of abound connection among different members. The more weak ties in the enterprises' network, the newer and the more unique information, which plays a very important role in S\&T innovation, can be easily acquired. As a result, the enterprises need construct the ties with government, intermediary agency, venture capitalist, supplier, and distributor, and bring these connections into the IUR network (Fig. 2). With these abound weak ties, members of the IUR can easily communicate the information, technology, and knowledge, and efficiently promote the transmission of S\&T achievements.

Besides, the property of network configuration is very important to acquire the information resources, and affects the efficiency of informational diffusion. With the difference from quantity of connection focused by the network connectivity, the network configuration concerns on quality and efficiency. According to the "structural holes" theory (Burt, 1992), most of networks of social relation are not often completely connected networks. In these networks, there are some structural holes (i.e., disconnections between irredundant contacts in a network), called by Burt. He noted that the dense network is inefficient in the sense that it returns less diverse information for the same cost as that of the sparse network. The enterprise with close linkage sometimes generates redundant information because of his redundant contacts. But the enterprise, occupying the structural holes, will get chance to contact the various heterogeneous and 
unrepeated information, which minimizes redundancy of information and generates information benefits. In addition, due to the position of structural holes, the structural holes also generate control benefits. Because the actor is in the structural holes and occupies the key route, he can create a bridge between otherwise disconnected members, and can decide the diffusion direction of various resource. Therefore, not the more and quick information but the position of the gaps between irredundant contacts, which gives certain players in a competitive arena an advantage in negotiating their relationships, is the most critical. Powell (1999) also thought that the enterprise being in the centre location of network can get more patents than other enterprises. Thus, the cooperative mechanism of IUR in which enterprise is in the core location should be established. The core enterprise should maintain the connection with other members, and create the bridge among other disconnected members to obtain much better network resources.

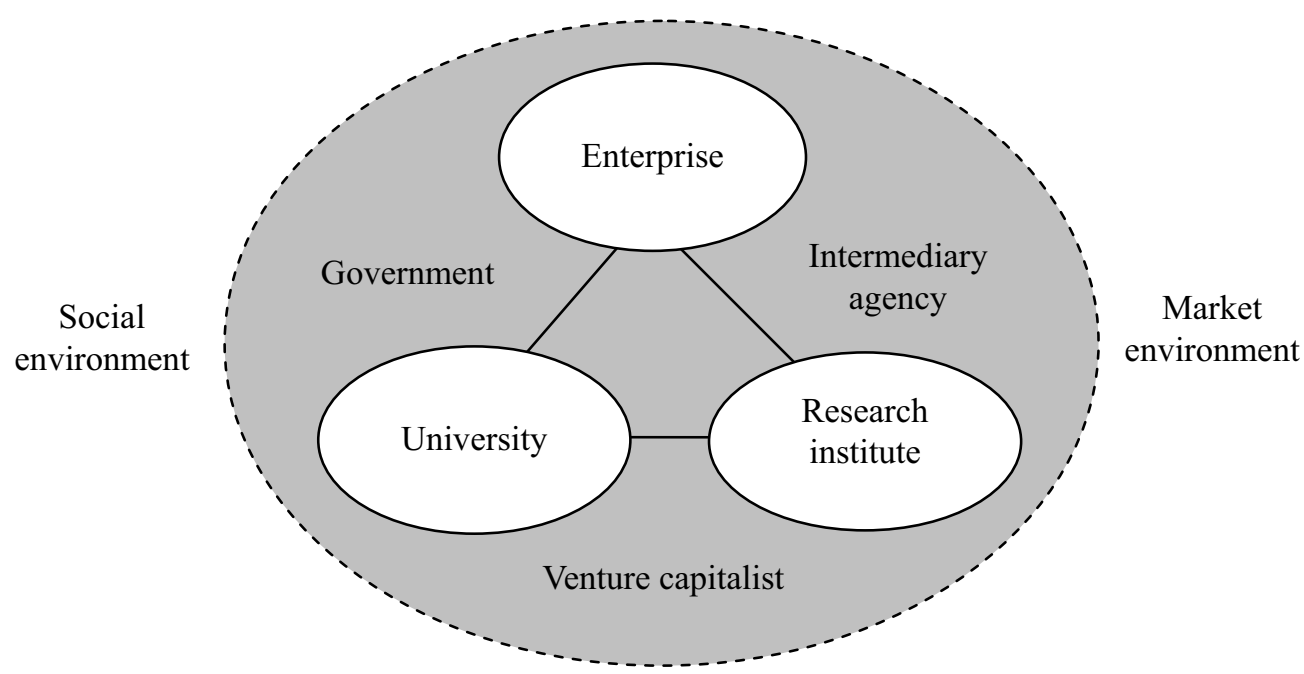

Figure 2. Structural dimension of the IUR social capital

\subsection{Analysis of relational dimension of the IUR social capital}

The relational dimension of social capital mainly refers to the trust and associability among organizations. The concepts of trust are inherently elusive and contested notions to the extent that they refer to highly complex forms of social relations and processes which are necessary for the generation and maintenance of collective action (Gambetta 1988; Giddens 1990; Kramer and Tyler 1996; Layder 1997). Thus, in the research of cooperative relationship, the inter-organizational trust is very important and critical (Lewicki, McAllister and Bies, 1998). Gulati (1995) thought that the inter-organizational trust can diminish the probability of opportunism of partners. The mutual trust derived from repeated connection in the IUR network should be the significant part of the cooperative governance mechanism. Because of replacing a part of contract, the trust thus reduces the costs of transactions produced in the IUR organization, and improves the performance of technological cooperation. Chiles and McMackin (1996) also pointed that relationships the high in trust, members are more willing to share and exchange the information. In the IUR network, the good cooperative relationship can produce trust between enterprises, universities and research institutes. Based on the trust and trustworthiness, they are more willing to participate in the cooperative technology innovation activities, and feel safer in developing the new products and technologies. Moreover, owing to the inter-organizational trust, they can effectively know each other, and frankly communicate. In fact, the trust promotes the development of cooperative creation, and enhances the enterprises' ability of absorbing knowledge (Scott, 2003). On the other hand, there is a two-way interaction between trust and cooperation: trust lubricates cooperation, and cooperation itself breeds trust. This may lead to the development, over time, of generalized norms of cooperation, which increase yet further the willingness to engage in social exchange and possibility of technological cooperation. Coleman (1988) suggests that "where a norm exists and is effective, it constitutes a powerful though sometimes fragile form of social capital".

The associability is also an important part in the relational dimension of social capital. Leana and Van Bure (1999) noted it as the degree of individual target obeying or associating with the organizational collective target. The inter-organizational social capital also has this property. The members of the IUR network not only exchange and combine the resource, knowledge and information, but also ensure their activities realizing their common target, in spite of individual interests. In the process of technological cooperation, the enterprise, the university and the research institute have different benefit aspirations, and they difficultly coordinate their goals. Because of the deviation between individual goal and collective goal, the incongruity of target often affects the success of the IUR cooperation. 


\subsection{Analysis of cognitive dimension of the IUR social capital}

The third dimension of social capital, which we label the "cognitive dimension," refers to those resources providing shared representations, interpretations, and systems of meaning among parties, including shared language and codes, and shared narratives. Nahapiet and Ghoshal (1997) noted that "to the extent that people share a common language, this facilitates their ability to gain access to people and their information. To the extent that their language and codes are different, this keeps people apart and restricts their access." Francis Fukuyama (1997) also indicated that "social capital ought to be simply formulated as a series of informal value and norm, with which individual and group are more willing to engage in cooperative interaction." Through the shared languages, codes, and values, the network members can easily communicate each other, decrease the coordination costs with the presence of misunderstanding, and diminish the probability of opportunism. Moreover, the shared language and codes are the filters of ideology, which provide the background and analytical framework for understanding and cognition, and facilitate the production of tacit knowledge. The realization of effective communication in the process of the IUR cooperation requires the similarly background of the members, which is the shared languages and representations of comprehension. Nevertheless, there are less shared and common systems of meaning because of the long-term completely different background of members. The enterprises specially concern on the productive technologies and the development of market, and pay attention to the economic benefit. In contrast, the universities and the research institutes are inclined to do the basic research, concern on the academic value of research, and are short of assurance to the market demand. The great difference in value, organizational routine, innovative consciousness, and organizational culture among them is the principal obstacle of tacit knowledge explicating and sticky knowledge transferring in the cooperative process. Therefore, the shared systems of meaning of the IUR, showed in the figure. 3 , ought to be constructed in the way of the tight connection and frequent communication.

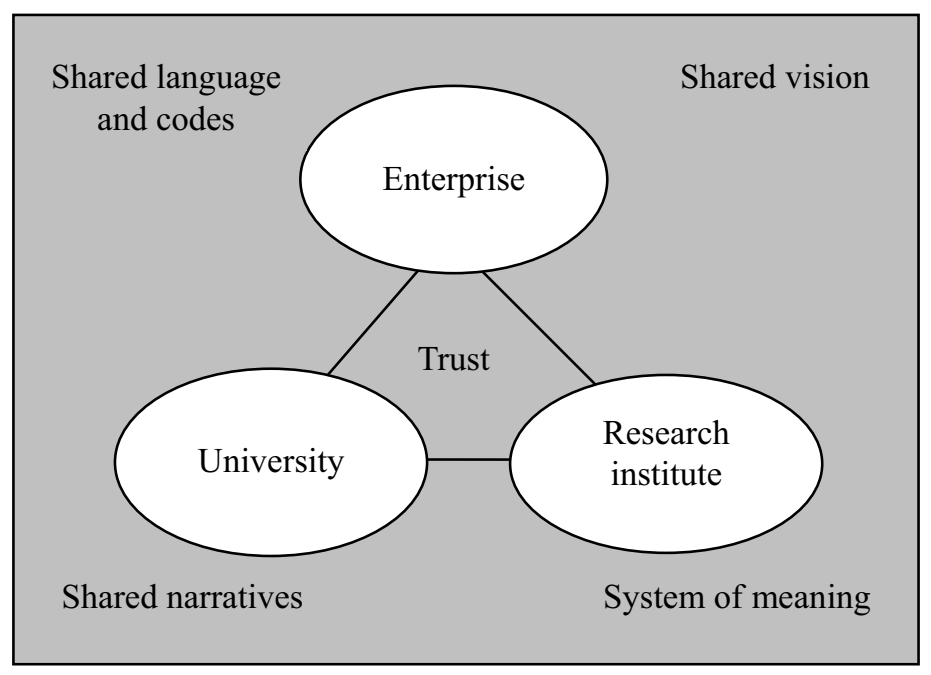

Figure 3. Relational and Cognitive dimension of the IUR social capital

\subsection{Analysis of relation of three dimensions of the IUR social capital}

There is difference between structural dimension, relational dimension and cognitive dimension. The structural dimension discusses whether there exits the network. The relational dimension discusses the quality of the network, especially focusing on the affective quality of relationship. However, the cognitive dimension concerns on the cognitive quality of relationship, for example whether truly comprehends each other or not (Bolino, 2002). Therefore, cognitive society capital is the deepest thing of the enterprise's social capital (Zhou xiao-hu, 2006). Besides, although social capital can be separated three dimensions analytically, they are actually inseparable. It is theoretically possible that structural social capital exists and cognitive social capital does not exist and vice versa, but it is practically impossible and difficult that one exists and another does not exist (Uphoff, 1999). It is difficult to imagine that there is a distinct and ordered network structure of a cooperative organization without shared language, shared codes and other shared systems of meaning. Conversely also the same, the groups with the shared systems of meaning can effectively cooperate with each other, without the relation among them. Finally, Tsai and Ghoshal (1998) thought that many of the features of social capital are highly interrelated. By the analysis of network data, they had preliminarily verified that both the structural and cognitive dimensions of social capital have a strong influence on the relational dimension; however the structural dimension of social capital has a weak influence on the cognitive dimension. Weiying (2007) indicated that the structural and relational dimensions can take effect with the cognitive dimension, but the cognitive dimension can effectively enhance the enterprises' ability of absorbing knowledge and even improve the innovation performance, with the high level of structural and relational dimensions. In fact, the three dimensions of social capital supplement and 
promote each other. The structural dimension of social capital, with the tight and extensive social connection of the IUR network, can reinforce the quality of trust, and further cultivate the shared narratives of cognitive dimension. Meanwhile, the cognitive dimension of social capital, with the shared value and vision, could realize the effective communication, form mutual trust of members, and strengthen the ties among network nodes. Trust of relational dimension social capital is able to enhance the tight connection, and cultivate and format the network culture of the IUR. Thus, in order to improve innovative performance, members of the IUR network should evenly increase the level of three dimensions of corporate social capital. And with their interrelation, they should positively improve the ability of the information communication and information acquirement to make success of the IUR cooperation.

\section{Conclusion and countermeasures}

Because of weakness of technological ability and insufficiency of resource for developing, the enterprise needs to acquire and accumulate information, knowledge and resource, and to manage them reasonably and effectively. In another word, that how to obtain the relevant information and technologies from the external social network, is the key set to solve the problems occurred in the enterprises' life. It requires the members of the IUR network, in the cultural atmosphere of tacit agreement and cooperation, to cultivate trust and collectivism, which forms the powerful social capital and supports sustainable development of the enterprise's innovation.

Based on the analysis of social capital of the IUR network, several steps must be made to successfully realize the IUR cooperation. Fist, we should establish the cooperative innovation networks of IUR, and enrich the weak ties of enterprises. On the one hand, the enterprise should use different patterns of IUR cooperation, such as technology transfer, joint development, joint-stock company, and project commission, to cultivate the strong ties of the IUR network, to promote communication of implicit knowledge and high quality information, and to decrease the uncertainty of obtaining information and opportunity cost. On the other hand, the enterprise should pay attention to enhance the weak ties with government, professional social intermediary institutions (e.g. consulting company, accounting firm, venture guiding center), suppliers, customers, venture capital investors, competitors and other external deliveries (e.g. guild, professional association, MBA club) to construct the opening network with technological cooperation orientation. Secondly, according to structure holes theory, the enterprise ought to concern about the strategic position in the cooperative innovation networks of IUR. They should actively construct the more beneficial network structures. That is to say that the enterprise should concentrate on developing the network structure holes or ally with the firm being in strategic position in the network to make itself approach the network centrality, and to gain the advantage resource thereby. Thirdly, the trust mechanism in the cooperative network should be constructed to form the shared systems of meaning. The members of the IUR should coordinate their different targets by molding the shared vision, and strengthen the effective communication to further reinforce the connection of network members. Correspondingly, the members should cultivate the mutual trust and cooperative network culture, and form the shared codes and shared narratives to diminish the obstacles of communication and propagation of tacit knowledge. Finally, the external innovation environment with the fundamental of social capital should be constructed. The government should guide and standard the construction process. The S\&T intermediate organizations oriented to the society should be established and perfected for technology diffusion, achievement transformation, technology evaluation, innovation decision, entrepreneurship decision and management consultation. The system of venture capital for the industrialization of S\&T be constructed and perfected to develop the diversified investment subjects, and to widen the channels of fund source. The public S\&T information platform should also be constructed, with the foundation of the S\&T information agency, the S\&T achievement agency, and the technological transaction institute, to transmit and communicate technical information in the wide area.

\section{References}

Baolin \& Huang long-xi. (2002). The Construction for the Middle-phase Links' of S\&T Achievements' Transformation. $R \& D$ Management, $4:$ 30-34.

Bourdieu P. (1985). The Forms of Capital. J G Richardson (Eds.), Handbook of Theory and Research for the Sociology of Education. New York: Greenwood Inc. pp: 241-258.

Burt, Ronald S (1992). Structural Holes: The Social Structure of Competition. Cambridge, MA: Harvard University Press.

Burt, Ronald S. (1997). The Contingent Valve of Social Capital. Administrative Science Quarterly, 42(2): 339-365.

Coleman J S. (1988). Social Capital In the Creation of Human Capital. American Journal of Sociology, 94(Supp.): S95-S120.

Fukuyama, F. (1997). Social Capital and the Modern Capitalist Economy: Creating a High Trust Workplace. Stern Business Magazine, 4(1).

Granovetter. M. S. (1973). The Strength of Weak Ties. American Journal of Sociology, 78: 1360-1380. 
Granovetter, M. S. (1985). Economic Action and Social Structure: The Problem of Embeddedness. American Journal of Sociology, 91: 481-510.

Gulati. (1995). Social Structure and Alliance Formation Patterns: A Longitudinal Analysis. Administrative Science Quarterly, 40: 619-652.

Guo Yi \& Zhu Xi. (2003). The New Development of Research of Foreign Social Capital and Management: Analytical Framework and Review of Application. Foreign Economies \& Management. 7: 2-7.

Leana C. \& Van Buren. (1999). Organizational Social Capital and Employment Practices. Academy of Management Review, 24(3): 538-555.

Lewicki R, McAllister D. \& Bies R. (1998). Trust and Distrust: New Relationships and Realities. Academy of Management Review, 23(3): 438-458.

Putnam R D. (1993). Making Democracy Work: Civic Traditions in Modern Italy. Princeton, NJ: Princeton University Press.

Nahapiet J, \& Ghoshal S. (1998). Social Capital, Intellectual Capital, and the Organizational Advantage. .Academy of Management Review, 23(2): 242-266.

Tsai W. \& Ghoshal S. (1998). Social Capital and Value Creation: the Role of Intrafirm Networks. Academy of Management Journal, 41: 464-478.

Saxenian A. (1991). The Origins and Dynamics of Production Networks in Silicon Valley. Research Policy, 20: 423-437.

Uphoff \& Norman. (1999). Understanding Social Capital: Learning From the Analysis and Experience of Participation. P. Dasgupta and I. Serageldin (Eds.) Social Capital: A Multifaceted Perspective. Washington, D.C.: The World Bank, pp: 215-249.

Wang Hua \& Lu Yan. (2007). The Research of Sustainable Competitive Advantage of Small and Medium-sized Enterprises In Perspective of Social Capital. Industrial Technology Economy. 5: 24-27.

Wei Ying. (2007). Corporate Social Capital and Technological Innovation: an Empirical Research Based on Perspective of Absorptive Capacity. China Industrial economy, 9: 119-127.

Wu Xiaobo, Wei Ying, \& Du Jian. (2004). The Analysis On the Effects of Social Capital In Industry-University-Research Institute Collaboration. Studies in Science of Science, 6: 630-633.

Zhou Xiaohu. (2006). Corporate social capital and strategy management. Beijing: People's Publishing House, (Chapter 3): $82-83$. 